\title{
Synthesis, Evaluation and Characterization of Some 1,3,4-Triazole-2-one Derivatives as Antimicrobial Agents
}

\author{
MAHABALESHWARAIAH NEELGUNDMATH ${ }^{\mathrm{a}, \mathrm{b}^{*}}$ AND \\ OBLENNAVAR KOTRESH ${ }^{\mathrm{b}}$
}

${ }^{a}$ Department of Chemistry, P. C. Jabin Science College, Hubli, Karnataka, India

${ }^{b}$ Department of Chemistry, Karnatak Science College, Dharwad, Karnataka, India

orgkotresh_org@rediffmail.com

Received 09 May 2011; Accepted 10 July 2011

\begin{abstract}
A series of novel compounds like 3[(phenyl substituted)-5methyl-1(Benzosulphonylamine)]-1,3,4-triazole-2-ones II(a-f) were synthesized by treating 4-amino-1-phenyl-3-methyl-5-oxo-1,2,4-triazoles with benzene sulphonyl chloride using pyridine as solvent. Similarly by using 4-amino-1-aryl-3-methyl-5-oxo-1,2,4-triazoles and acetic anhydride as starting material 3[(phenyl substituted)-5-methyl-1(acetylamino)]1,3,4-triazole-2-ones III(a-f) were synthesized and also 3[(phenyl substituted)-5-methyl-1(chloroacetyl)]-1,3,4-triazole-2-ones I(a-f) were synthesized by treating 4-amino-1-aryl-3-methyl-5-oxo-1,2,4-triazoles with chloroacetyl chloride in presence of a non-polar solvent like benzene. Elemental analysis, GCMS, IR, and ${ }^{1} \mathrm{H}$ NMR confirmed the structures of the newly synthesised compounds. The newly synthesized compounds are also screened for their antibacterial, antifungal and antiinflammatory activities.
\end{abstract}

Keywords: 4-amino-1-phenyl-3-methyl-5-oxo-1,2,4-triazoles, benzene sulphonyl chloride, antibacterial, antifungal \& anti-inflammatory activities.

\section{Introduction}

4-amino-1-phenyl-3-methyl-5-oxo-1,2,4-triazole have been consistently rewarded as a promising molecule because of its broad spectrum pharmaceutical activity like antibacterial $^{1}$, antifungal ${ }^{2}$, and moderate anti-inflammatory activities ${ }^{3}$.

The triazoles were synthesized from sydnones as starting material were mesoionic in nature (meso + ionic). The aromaticity of these compounds can be explained based on the classical sextet theory. There are a total of seven $2 \mathrm{p}_{\mathrm{z}}$ - electrons supplied by the atoms which makeup the ring to have sextet electrons in the ring. The yield of triazoles is nearly $75 \%$. Triazoles are potent heterocyclic compounds having antibacterial and antifungal activities.

$3[($ phenyl substituted)-5-methyl-1(Benzosulphonylamine)]-1,3,4-triazole-2-ones[II(af)] were synthesized by treating 4-amino-1-aryl-3-methyl-5-oxo-1,2,4-triazoles with chloroacetyl chloride in benzene sulphonyl chloride using pyridine as solvent. Similarly by using 4-amino-1-aryl-3-methyl-5-oxo-1,2,4-triazoles and acetic anhydride as starting 
material the compounds $3[$ (phenyl substituted)-5-methyl-1(acetylamino)]-1,3,4-triazole-2ones[III(a-f)] and also 3[(phenyl substituted)-5-methyl-1(chloroacetyl)]-1,3,4-triazole-2ones $[\mathrm{I}(\mathrm{a}-\mathrm{f})]$ is synthesized by treating 4-amino-1-aryl-3-methyl-5-oxo-1,2,4-triazoles in presence of a non-polar solvent like benzene. All synthesized heterocycles are showing prominent antibacterial, antifungal and moderate anti-inflammatory activity.

$3[$ (phenyl substituted)-5-methyl-1(chloroacetylamine)]-1,3,4-triazole-2-ones [I(a-f)] are more antifungal and antibacterial in nature, which are synthesized by using 4-amino-1-aryl3-methyl-5-oxo-1,2,4-triazoles with chloroacetyl chloride in presence of benzene as non polar solvent. (Scheme-1)

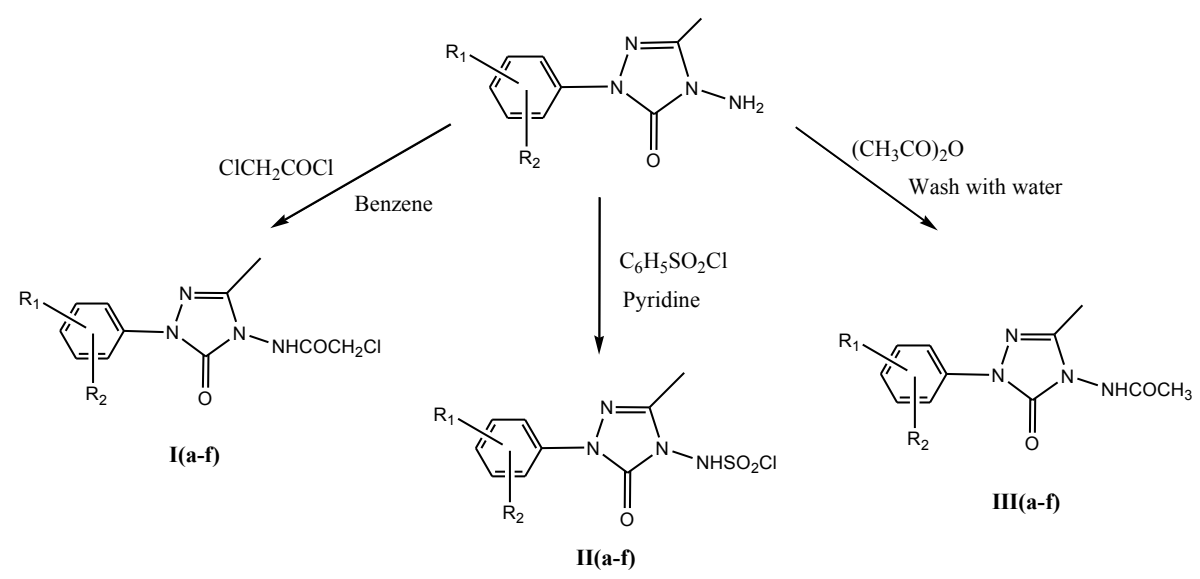

Scheme 1

The IR spectra of I(a-f) showed characteristic absorption band at $1680 \mathrm{~cm}^{-1}$ is due to $>\mathrm{C}=\mathrm{O}$ group of side chain and adsorption band at $1722 \mathrm{~cm}^{-1}$ is due to another ring $>\mathrm{C}=\mathrm{O}$ group. The $\mathrm{NH}_{2}$ peak appeared in triazoles is disappeared by the formation of 3[(phenyl substituted)-5-methyl-1(chloroacetylamine)]-1,3,4-triazole-2-ones I(a-f). The -NH group in the product is appeared at $3002 \mathrm{~cm}^{-1}$.

In II(a-f), the IR band corresponds to $-\mathrm{NH}$ is appeared at $2932 \mathrm{~cm}^{-1}$, the $-\mathrm{S}=\mathrm{O}$ is appearing at $1491 \mathrm{~cm}^{-1}$, similarly in $\mathrm{III}(\mathrm{a}-\mathrm{f})$ products the $>\mathrm{C}=\mathrm{O}$ stretching of amide is at $1548 \mathrm{~cm}^{-1}$.

The ${ }^{1} \mathrm{H}$ NMR, LCMS, IR and elemental analysis supported the structure of title compounds. Physical and analytical data of title compound and its other substituted derivatives are given in Table 1, Table 2 and Table 3.

\section{Experimental}

All the chemicals were purchased from Merck and used without purification. Analytical TLC was performed on silica Gel $\mathrm{F}_{254}$ plates (Merck) with visualization by UV light. Melting points were determined in open capillaries on a Thermonik melting point apparatus, Mumbai, India and are uncorrected. The IR spectra $\left(\mathrm{KBr}, \gamma \max , \mathrm{cm}^{-1}\right)$ were run on Shimadzu-8400 FTIR spectrophotometer. ${ }^{1} \mathrm{H}$ NMR $\left(\delta \mathrm{ppm}, \mathrm{CDCl}_{3} / \mathrm{DMSO}-\mathrm{d}_{6}\right)$ spectra were recorded using Brucker WM-400 spectrometer with TMS as internal standard. Mass spectra 
were recorded on Micromass Q-TOF and Shimadzu LCMS 2010A Mass spectrometer. Elemental analysis was performed on Thermo Finnigam FLASH EA 1122 CHNS analyzer and was within $0.4 \%$ theoretical values.

Synthesis of 3[(phenyl substituted)-5-methyl-1(chloroacetylamine)]-1,3,4-triazole-2ones $[I(a-f)]$

4-amino-1-aryl-3-methyl-5-oxo-1,2,4-triazole $(0.1 \mathrm{~mol})$ and chloro acetyl chloride $(0.1 \mathrm{~mol})$ is taken in round bottom flask to this $100 \mathrm{~mL}$ of benzene is added and made homogenous by stirring on a magnetic stirrer for $45 \mathrm{~min}$. After the completion of reaction the solution is poured on crushed ice and obtained solid was filtered and dried. The product was recrystallized from absolute alcohol. Yield: 75\%; M.P: $125-129^{0} \mathrm{C}$.

The IR spectra of I(a-f) showed characteristic absorption band at $1680 \mathrm{~cm}^{-1}$ is due to $>\mathrm{C}=\mathrm{O}$ group of side chain and adsorption band at $1722 \mathrm{~cm}^{-1}$ is due to another ring $>\mathrm{C}=\mathrm{O}$ group. The $\mathrm{NH}_{2}$ peak appeared in triazoles is disappeared by the formation of $3[$ (phenyl substituted)-5-methyl-1(chloroacetylamine)]-1,3,4-triazole-2-ones I(a-f). The -NH group in the product is appeared at $3002 \mathrm{~cm}^{-1}$.

Table 1. Physical and analytical data of compound I and its other substituted derivatives.

\begin{tabular}{|c|c|c|c|c|c|}
\hline Compund & $\mathrm{R}_{1}$ & $\mathrm{R}_{2}$ & Molecular Formula & Yield, \% & Melting Point, ${ }^{\circ} \mathrm{C}$ \\
\hline $\mathrm{Ia}$ & $4 \mathrm{Cl}$ & $2 \mathrm{Cl}$ & $\mathrm{C}_{10} \mathrm{H}_{7} \mathrm{~N}_{4} \mathrm{O}_{2} \mathrm{Cl}_{3}$ & 45 & $245-247$ \\
\hline $\mathrm{Ib}$ & $4 \mathrm{CH}$ & $2 \mathrm{Cl}$ & $\mathrm{C}_{11} \mathrm{H}_{10} \mathrm{~N}_{4} \mathrm{O}_{2} \mathrm{Cl}_{2}$ & 58 & $285-287$ \\
\hline $\mathrm{Ic}$ & $4 \mathrm{Br}$ & $2 \mathrm{CH}_{3}$ & $\mathrm{C}_{11} \mathrm{H}_{10} \mathrm{~N}_{4} \mathrm{O}_{2} \mathrm{ClBr}$ & 42 & $203-204$ \\
\hline $\mathrm{Id}$ & $\mathrm{H}$ & $\mathrm{H}$ & $\mathrm{C}_{10} \mathrm{H}_{9} \mathrm{~N}_{4} \mathrm{O}_{2} \mathrm{Cl}$ & 40 & $320-322$ \\
\hline $\mathrm{Ie}$ & $4 \mathrm{OCH}_{3}$ & $\mathrm{H}$ & $\mathrm{C}_{11} \mathrm{H}_{11} \mathrm{~N}_{4} \mathrm{O}_{3} \mathrm{Cl}$ & 52 & $298-300$ \\
\hline If & $4 \mathrm{OCH}_{3}$ & $2 \mathrm{OCH}_{3}$ & $\mathrm{C}_{12} \mathrm{H}_{13} \mathrm{~N}_{4} \mathrm{O}_{4} \mathrm{Cl}$ & 40 & $292-294$ \\
\hline
\end{tabular}

Synthesis of 3[(phenyl substituted)-5-methyl-1(benzosulphonylamine)]-1,3,4-triazole-2ones $[I I(a-f)]$

4-amino-1-aryl-3-methyl-5-oxo-1,2,4-triazole ( $0.1 \mathrm{~mol})$ and benzene sulphonyl chloride $(0.1$ $\mathrm{mol})$ in pyridine $(40 \mathrm{ml})$ placed in a round bottomed flask. The reaction mixture was stirred at reflux temperature for $1 \mathrm{~h}$. After the completion of the reaction, the mixture is added in ice cold water. The residue obtained was treated with a $5 \%$ solution of $\mathrm{Na}_{2} \mathrm{CO}_{3}(100 \mathrm{~mL})$, the obtained solid was filtered, washed with water, dried and recrystallized with suitable solvent.

In II(a-f), the IR band corresponds to $-\mathrm{NH}$ is appeared at $2932 \mathrm{~cm}^{-1}$, the $-\mathrm{S}=\mathrm{O}$ is appearing at $1491 \mathrm{~cm}^{-1}$.

Table 2. Physical and analytical data of compound II and its other substituted derivatives.

\begin{tabular}{|c|c|c|l|c|c|}
\hline Compund & $\mathrm{R}_{1}$ & $\mathrm{R}_{2}$ & \multicolumn{1}{|c|}{ Molecular Formula } & Yield, \% & $\begin{array}{c}\text { Melting Point, } \\
{ }^{0} \mathrm{C}\end{array}$ \\
\hline IIa & $4 \mathrm{Cl}$ & $2 \mathrm{Cl}$ & $\mathrm{C}_{15} \mathrm{H}_{12} \mathrm{~N}_{4} \mathrm{O}_{3} \mathrm{SCl}_{2}$ & 42 & $265-267$ \\
\hline IIb & $4 \mathrm{CH}_{3}$ & $2 \mathrm{Cl}$ & $\mathrm{C}_{16} \mathrm{H}_{15} \mathrm{~N}_{4} \mathrm{O}_{3} \mathrm{SCl}$ & 52 & $305-307$ \\
\hline IIc & $4 \mathrm{Br}$ & $2 \mathrm{CH}_{3}$ & $\mathrm{C}_{16} \mathrm{H}_{15} \mathrm{~N}_{4} \mathrm{O}_{3} \mathrm{SBr}$ & 39 & $223-224$ \\
\hline IId & $\mathrm{H}$ & $\mathrm{H}$ & $\mathrm{C}_{15} \mathrm{H}_{14} \mathrm{~N}_{4} \mathrm{O}_{3} \mathrm{~S}$ & 43 & $340-342$ \\
\hline IIe & $4 \mathrm{OCH}_{3}$ & $\mathrm{H}$ & $\mathrm{C}_{16} \mathrm{H}_{16} \mathrm{~N}_{4} \mathrm{O}_{4} \mathrm{~S}$ & 54 & $308-310$ \\
\hline IIf & $4 \mathrm{OCH}_{3}$ & $2 \mathrm{OCH}_{3}$ & $\mathrm{C}_{17} \mathrm{H}_{18} \mathrm{~N}_{4} \mathrm{O}_{5} \mathrm{~S}$ & 48 & $320-324$ \\
\hline
\end{tabular}


Synthesis of 3[(phenyl substituted)-5-methyl-1(acetylamino)]-1,3,4-triazole-2-ones [III( $(a-f)]$

A mixture of 4-amino-1-aryl-3-methyl-5-oxo-1, 2, 4-triazole $(0.1 \mathrm{~mol})$ and acetic anhydride $(0.01 \mathrm{~mol}$.) $25 \mathrm{ml}$ is taken in a round-bottomed flask and refluxed for 2 to 3 hours. After the completion of the reaction the contents of the flask is poured on crushed ice, the solid separated was filtered and dried. The product was recrystallized with suitable solvents.

In compounds III(a-f), the IR band corresponding to the $>\mathrm{C}=\mathrm{O}$ stretching of amide is at $1548 \mathrm{~cm}^{-1}$ and $-\mathrm{NH}$ stretching is appeared at $3010 \mathrm{~cm}^{-1}$.

Table 3. Physical and analytical data of compound III and its other substituted derivatives.

\begin{tabular}{|c|c|c|l|c|c|}
\hline Compund & $\mathrm{R}_{1}$ & $\mathrm{R}_{2}$ & \multicolumn{1}{|c|}{ Molecular Formula } & Yield, \% & Melting Point, ${ }^{0} \mathrm{C}$ \\
\hline IIIa & $4 \mathrm{Cl}$ & $2 \mathrm{Cl}$ & $\mathrm{C}_{11} \mathrm{H}_{10} \mathrm{~N}_{4} \mathrm{O}_{2} \mathrm{Cl}_{2}$ & 46 & $255-257$ \\
\hline IIIb & $4 \mathrm{CH}_{3}$ & $2 \mathrm{Cl}$ & $\mathrm{C}_{12} \mathrm{H}_{13} \mathrm{~N}_{4} \mathrm{O}_{2} \mathrm{Cl}$ & 59 & $295-297$ \\
\hline IIIc & $4 \mathrm{Br}$ & $2 \mathrm{CH}_{3}$ & $\mathrm{C}_{12} \mathrm{H}_{13} \mathrm{~N}_{4} \mathrm{O}_{2} \mathrm{Br}$ & 45 & $213-215$ \\
\hline IIId & $\mathrm{H}$ & $\mathrm{H}$ & $\mathrm{C}_{11} \mathrm{H}_{12} \mathrm{~N}_{4} \mathrm{O}_{2}$ & 41 & $330-332$ \\
\hline IIIe & $4 \mathrm{OCH}_{3}$ & $\mathrm{H}$ & $\mathrm{C}_{12} \mathrm{H}_{14} \mathrm{~N}_{4} \mathrm{O}_{2}$ & 51 & $301-303$ \\
\hline IIIf & $4 \mathrm{OCH}_{3}$ & $2 \mathrm{OCH}_{3}$ & $\mathrm{C}_{13} \mathrm{H}_{16} \mathrm{~N}_{4} \mathrm{O}_{4}$ & 42 & $312-314$ \\
\hline
\end{tabular}

3[(2,4-dichloro phenyl)-5-methyl-1(chloroacetyl)]-1,3,4-triazole-2-ones (Ia)

IR $\left(\mathrm{KBr}, \mathrm{cm}^{-1}\right): 1680(>\mathrm{C}=\mathrm{O}$ Stretching of side chain), 1722 (ring $>\mathrm{C}=\mathrm{O}$ Stretching) \& 3045 $\mathrm{cm}^{-1}$ (NH stretching). ${ }^{1} \mathrm{H}$ NMR (DMSO-d $\left.\mathrm{d}_{6}\right) \delta: 6.6-8.1(\mathrm{~m}, 3 \mathrm{H}, \mathrm{Ar}-\mathrm{H}), 2.20\left(\mathrm{~s}, 3 \mathrm{H}, \mathrm{N}-\mathrm{CH}_{3}\right)$. $8.2(\mathrm{~s}, 1 \mathrm{H},-\mathrm{NH})$. Mass spectra $(\mathrm{m} / \mathrm{z}): \mathrm{M}^{+1}=320$. Elemental data cal, $\mathrm{C}, 37.35 ; \mathrm{H}, 2.19 ; \mathrm{N}$, 17.42; Cl, 33.08; O, 9.95. Found: C, 37.05; H, 2.09; N, 17.32; Cl, 32.98; O, 9.85. Yield: 45\%. MP: $245-247^{\circ} \mathrm{C}$.

3[(2-methyl, 4-chloro phenyl)-5-methyl-1(chloroacetyl)]-1,3,4-triazole-2-ones (Ib) IR $\left(\mathrm{KBr}, \mathrm{cm}^{-1}\right): 1680(>\mathrm{C}=\mathrm{O}$ Stretching of side chain), 1722 (ring $>\mathrm{C}=\mathrm{O}$ Stretching) \& 3045 $\mathrm{cm}^{-1}$ (NH stretching). ${ }^{1} \mathrm{H}$ NMR (DMSO-d $\left.\mathrm{d}_{6}\right) \delta: 6.6-8.2(\mathrm{~m}, 3 \mathrm{H}, \mathrm{Ar}-\mathrm{H}), 2.20\left(\mathrm{~s}, 3 \mathrm{H}, \mathrm{N}-\mathrm{CH}_{3}\right)$, $5.48\left(\mathrm{~s}, 3 \mathrm{H}, \mathrm{CH}_{3}\right), 8.2(\mathrm{~s}, 1 \mathrm{H},-\mathrm{NH})$. Mass spectra $(\mathrm{m} / \mathrm{z}): \mathrm{M}^{+1}=300$. Elemental data cal, $\mathrm{C}$, 43.87; H, 3.35; N, 18.61; Cl, 23.55; O, 10.63. Found: C, 43.77; H, 3.27; N, 18.51; Cl, 23.45; O, 10.55. Yield: $58 \%$. MP: $285-287^{\circ} \mathrm{C}$.

3[(2-bromo, 4-methyl phenyl)-5-methyl-1(chloroacetyl)]-1,3,4-triazole-2-ones (Ic)

IR $\left(\mathrm{KBr}, \mathrm{cm}^{-1}\right): 1680(>\mathrm{C}=\mathrm{O}$ Stretching of side chain), 1722 (ring $>\mathrm{C}=\mathrm{O}$ Stretching) \& 3045 $\mathrm{cm}^{-1}$ (NH stretching). ${ }^{1} \mathrm{H}$ NMR (DMSO-d $\left.\mathrm{d}_{6}\right) \delta: 6.6-8.2(\mathrm{~m}, 3 \mathrm{H}, \mathrm{Ar}-\mathrm{H}), 2.20\left(\mathrm{~s}, 3 \mathrm{H}, \mathrm{N}-\mathrm{CH}_{3}\right.$ ), $5.48\left(\mathrm{~s}, 3 \mathrm{H}, \mathrm{CH}_{3}\right), 8.2(\mathrm{~s}, 1 \mathrm{H},-\mathrm{NH})$. Mass spectra $(\mathrm{m} / \mathrm{z}): \mathrm{M}^{+1}=344$. Elemental data cal, $\mathrm{C}$, 38.23; H, 2.92; N, 16.21; Cl, 10.26; Br, 23.12; O, 9.26. Found: C, 38.15; H, 2.84; N, 16.11; $\mathrm{Cl}, 10.14$; Br, 23.04; O, 9.16. Yield: 42\%. MP: $203-204{ }^{\circ} \mathrm{C}$.

3[(phenyl)-5-methyl-1(chloroacetyl)]-1,3,4-triazole-2-ones (Id)

IR $\left(\mathrm{KBr}, \mathrm{cm}^{-1}\right)$ : $1680(>\mathrm{C}=\mathrm{O}$ Stretching of side chain), 1722 (ring $>\mathrm{C}=\mathrm{O}$ Stretching) \& 3045 $\mathrm{cm}^{-1}$ (NH stretching). ${ }^{1} \mathrm{H}$ NMR (DMSO-d $\left.\mathrm{d}_{6}\right) \delta: 6.0-8.8(\mathrm{~m}, 5 \mathrm{H}, \mathrm{Ar}-\mathrm{H}), 2.20\left(\mathrm{~s}, 3 \mathrm{H}, \mathrm{N}-\mathrm{CH}_{3}\right.$ ), $8.2(\mathrm{~s}, 1 \mathrm{H},-\mathrm{NH})$. Mass spectra $(\mathrm{m} / \mathrm{z}): \mathrm{M}^{+1}=252$. Elemental data cal, $\mathrm{C}, 47.54 ; \mathrm{H}, 3.59 ; \mathrm{N}$, 22.18; Cl, 14.03; O, 12.66. Found: C, 47.44; H, 3.47; N, 22.11; Cl, 13.95; O, 12.58. Yield: 40\%. MP: 320- 322 oC.

3[(4-methoxy phenyl)-5-methyl-1(chloroacetyl)]-1,3,4-triazole-2-ones (Ie)

IR $\left(\mathrm{KBr}, \mathrm{cm}^{-1}\right): 1680(>\mathrm{C}=\mathrm{O}$ Stretching of side chain), 1722 (ring $>\mathrm{C}=\mathrm{O}$ Stretching) \& 3045 $\mathrm{cm}^{-1}$ (NH stretching). ${ }^{1} \mathrm{H}$ NMR (DMSO-d ${ }_{6}$ ) $\delta: 6.6-8.2(\mathrm{~m}, 4 \mathrm{H}, \mathrm{Ar}-\mathrm{H}), 2.20$ (s, 3H, N-CH ${ }_{3}$ ), 
$3.68\left(\mathrm{~s}, 3 \mathrm{H}, 4 \mathrm{OCH}_{3}\right), 8.2(\mathrm{~s}, 1 \mathrm{H},-\mathrm{NH})$. Mass spectra $(\mathrm{m} / \mathrm{z}): \mathrm{M}^{+1}=282$. Elemental data cal, C, 46.74; H, 3.92; Cl, 12.54; N,19.82; O, 16.98. Found: C, 46.66; H, 3.84; Cl, 12.46; N,19.77; O, 16.88. Yield: $52 \%$. MP: $298-300^{\circ} \mathrm{C}$.

3[(2, 4-dimethoxy phenyl)-5-methyl-1(chloroacetyl)]-1,3,4-triazole-2-ones (If) IR $\left(\mathrm{KBr}, \mathrm{cm}^{-1}\right): 1680(>\mathrm{C}=\mathrm{O}$ Stretching of side chain), 1722 (ring $>\mathrm{C}=\mathrm{O}$ Stretching) \& 3045 $\mathrm{cm}^{-1}$ (NH stretching). ${ }^{1} \mathrm{H}$ NMR (DMSO-d $\left.{ }_{6}\right) \delta: 6.6-8.2(\mathrm{~m}, 4 \mathrm{H}, \mathrm{Ar}-\mathrm{H}), 2.20\left(\mathrm{~s}, 3 \mathrm{H}, \mathrm{N}-\mathrm{CH}_{3}\right)$, $3.78\left(\mathrm{~s}, 6 \mathrm{H}, 2 \mathrm{OCH}_{3}, 4 \mathrm{OCH}_{3}\right), 8.2(\mathrm{~s}, 1 \mathrm{H},-\mathrm{NH})$. Mass spectra $(\mathrm{m} / \mathrm{z}): \mathrm{M}^{+1}=312$. Elemental data cal, C, 46.09; H, 4.19; Cl, 11.34; N, 17.92; O, 20.47. Found: C, 46.02; H, 4.12; Cl, 11.26; N, 17.84; O, 20.41. Yield: 40\%. MP: $292-294^{\circ} \mathrm{C}$.

3[(2,4-dichlro phenyl)-5-methyl-1benzosulphonylamine)]-1,3,4-triazole-2-ones (IIa)

IR $\left(\mathrm{KBr}, \mathrm{cm}^{-1}\right)$ : 1724 (ring $>\mathrm{C}=\mathrm{O}$ Stretching), $1491\left(-\mathrm{S}=\mathrm{O}\right.$ stretching) \& $2932 \mathrm{~cm}^{-1}(\mathrm{NH}$ stretching). ${ }^{1} \mathrm{H}$ NMR (DMSO-d $\left.{ }_{6}\right) \delta: 6.2-8.9(\mathrm{~m}, 8 \mathrm{H}, \mathrm{Ar}-\mathrm{H}), 2.22\left(\mathrm{~s}, 3 \mathrm{H}, \mathrm{N}-\mathrm{CH}_{3}\right), 8.2(\mathrm{~s}, 1 \mathrm{H},-$ $\mathrm{NH})$. Mass spectra $(\mathrm{m} / \mathrm{z}): \mathrm{M}^{+1}=398$. Elemental data cal, C, 45.12; H, 3.03; Cl, 17.76; N, 14.03; O, 12.02; S, 8.03. Found: C, 45.06; H, 2.93; Cl, 17.68; N, 13.96; O,11.94; S, 7.97. Yield: $42 \%$. MP: $265-267^{\circ} \mathrm{C}$.

3[(2-methyl, 4-chloro phenyl)-5-methyl-1benzosulphonylamine)]-1,3,4-triazole-2-ones (IIb) IR $\left(\mathrm{KBr}, \mathrm{cm}^{-1}\right)$ : 1724 (ring $>\mathrm{C}=\mathrm{O}$ Stretching), 2913 (C-H stretching), 1491 (-S=O stretching) $\& 2932 \mathrm{~cm}^{-1}$ (NH stretching). ${ }^{1} \mathrm{H}$ NMR $\left(\mathrm{DMSO}_{-} \mathrm{d}_{6}\right) \delta: 6.2-8.9(\mathrm{~m}, 8 \mathrm{H}, \mathrm{Ar}-\mathrm{H}), 2.22(\mathrm{~s}, 3 \mathrm{H}, \mathrm{N}-$ $\left.\mathrm{CH}_{3}\right), 4.12\left(\mathrm{~s}, 3 \mathrm{H}, \mathrm{CH}_{3}\right), 8.2(\mathrm{~s}, 1 \mathrm{H},-\mathrm{NH})$. Mass spectra $(\mathrm{m} / \mathrm{z}): \mathrm{M}^{+1}=378$. Elemental data cal, C, 50.73; H, 3.99; Cl, 9.36; N, 14.79; O,12.67; S, 8.46. Found: C, 50.64; H, 3.87; Cl, 9.28; N, 14.68; O,12.56; S, 8.42. Yield: $42 \%$. MP: $305-307^{\circ} \mathrm{C}$.

3[(2-methyl, 4-bromo phenyl)-5-methyl-1benzosulphonylamine)]-1,3,4-triazole-2-ones (IIc) IR $\left(\mathrm{KBr}, \mathrm{cm}^{-1}\right)$ : 1724 (ring $>\mathrm{C}=\mathrm{O}$ Stretching), 2913 (C-H stretching), 1491 (-S=O stretching) $\& 2932 \mathrm{~cm}^{-1}$ (NH stretching). ${ }^{1} \mathrm{H}$ NMR $\left(\right.$ DMSO-d $\left._{6}\right) \delta: 6.2-8.9(\mathrm{~m}, 8 \mathrm{H}, \mathrm{Ar}-\mathrm{H}), 2.22(\mathrm{~s}, 3 \mathrm{H}, \mathrm{N}-$ $\left.\mathrm{CH}_{3}\right), 4.12\left(\mathrm{~s}, 3 \mathrm{H}, \mathrm{CH}_{3}\right), 8.2(\mathrm{~s}, 1 \mathrm{H},-\mathrm{NH})$. Mass spectra $(\mathrm{m} / \mathrm{z}): \mathrm{M}^{+1}=424$. Elemental data cal, C, 45.40; H, 3.57; Br, 18.88; N, 13.24; O, 11.34; S, 7.58. Found: C, 45.22; H, 3.37; Br, 18.68; N, 13.02; O,11.14; S, 7.36.Yield: 39\%. MP: $223-224^{\circ} \mathrm{C}$.

3[(phenyl)-5-methyl-1benzosulphonylamine)]-1,3,4-triazole-2-ones (IId)

IR $\left(\mathrm{KBr}, \mathrm{cm}^{-1}\right)$ : 1722 (ring $>\mathrm{C}=\mathrm{O}$ Stretching) \& $3045 \mathrm{~cm}^{-1}$ (NH stretching). ${ }^{1} \mathrm{H}$ NMR $\left(\mathrm{DMSO}_{\mathrm{d}}\right)$ $)$ : 6.0-8.9 (m, 10H, Ar-H), $2.20\left(\mathrm{~s}, 3 \mathrm{H}, \mathrm{N}-\mathrm{CH}_{3}\right), 8.2(\mathrm{~s}, 1 \mathrm{H},-\mathrm{NH})$. Mass spectra $(\mathrm{m} / \mathrm{z}): \quad \mathrm{M}^{+1}=315$. Elemental data cal, $\mathrm{C}, 57.31 ; \mathrm{H}, 4.49 ; \mathrm{N}, 17.82 ; \mathrm{O}, 10.18 ; \mathrm{S}, 10.20$. Found: C, 57.12; H, 4.28; N, 17.62; O, 10.02; S, 10.02.Yield: 43\%. MP: $340-342^{\circ} \mathrm{C}$.

3[(4-methoxy phenyl)-5-methyl-1benzosulphonylamine)]-1,3,4-triazole-2-ones (IIe) IR $\left(\mathrm{KBr}, \mathrm{cm}^{-1}\right)$ : 1722 (ring $>\mathrm{C}=\mathrm{O}$ Stretching) \& $3045 \mathrm{~cm}^{-1}$ (NH stretching). ${ }^{1} \mathrm{H}$ NMR $\left(\mathrm{DMSO}_{-} \mathrm{d}_{6}\right) \delta: 6.0-8.9(\mathrm{~m}, 10 \mathrm{H}, \mathrm{Ar}-\mathrm{H}), 2.20\left(\mathrm{~s}, 3 \mathrm{H}, \mathrm{N}-\mathrm{CH}_{3}\right), 3.81\left(\mathrm{~s}, 3 \mathrm{H}, \mathrm{OCH}_{3}\right), 8.2(\mathrm{~s}, 1 \mathrm{H}$, -NH). Mass spectra $(\mathrm{m} / \mathrm{z}): \mathrm{M}^{+1}=360$. Elemental data cal,. C, 53.32; H, 4.47; N, 15.50; O, 17.76; S, 8.90. Found: C, 53.20; H, 4.42; N, 15.46; O, 17.69; S, 8.88. Yield: 54\%. MP: 308$310^{\circ} \mathrm{C}$.

3[(2,4-dimethoxy phenyl)-5-methyl-1benzosulphonylamine)]-1,3,4-triazole-2-ones (IIf) IR $\left(\mathrm{KBr}, \mathrm{cm}^{-1}\right): 1722$ (ring $>\mathrm{C}=\mathrm{O}$ Stretching) \& $3045 \mathrm{~cm}^{-1}$ ( $\mathrm{NH}$ stretching). ${ }^{1} \mathrm{H}$ NMR $\left(\mathrm{DMSO}_{\mathrm{d}}\right)$ ) $8: 6.0-8.9(\mathrm{~m}, 10 \mathrm{H}, \mathrm{Ar}-\mathrm{H}), 2.20\left(\mathrm{~s}, 3 \mathrm{H}, \mathrm{N}-\mathrm{CH}_{3}\right), 3.81\left(\mathrm{~s}, 6 \mathrm{H}, \mathrm{OCH}_{3}\right), 8.2(\mathrm{~s}, 1 \mathrm{H}$, -NH). Mass spectra $(\mathrm{m} / \mathrm{z}): \mathrm{M}^{+1}=390$. Elemental data cal, C, 52.30; H, 4.65; N, 14.35; O, 
20.49; S, 8.21. Found: C, 52.20; H, 4.45; N, 14.38; O, 20.42; S, 8.24. Yield: 48\%. MP: 320$324{ }^{\circ} \mathrm{C}$.

3[(2,4-dichlro phenyl)-5-methyl-1acetylamino)]-1,3,4-triazole-2-ones (IIIa)

IR $\left(\mathrm{KBr}, \mathrm{cm}^{-1}\right): 1684(>\mathrm{C}=\mathrm{O}$ Stretching of side chain), 1726 (ring $>\mathrm{C}=\mathrm{O}$ Stretching), \& 2932 $\mathrm{cm}^{-1}$ (NH stretching). ${ }^{1} \mathrm{H}$ NMR (DMSO-d $\left.{ }_{6}\right) \delta: 6.2-8.9(\mathrm{~m}, 3 \mathrm{H}, \mathrm{Ar}-\mathrm{H}), 2.22\left(\mathrm{~s}, 3 \mathrm{H}, \mathrm{N}_{-} \mathrm{CH}_{3}\right)$, $8.2(\mathrm{~s}, 1 \mathrm{H},-\mathrm{NH})$. Mass spectra $(\mathrm{m} / \mathrm{z}): \mathrm{M}^{+1}=300$. Elemental data cal, $\mathrm{C}, 43.87 ; \mathrm{H}, 3.35 ; \mathrm{Cl}$, 23.55; N, 18.61; O, 10.63. Found: C, 43.89; H, 3.32; Cl, 23.51; N, 18.64; O, 10.53. Yield: 46\%. MP: $255-257^{\circ} \mathrm{C}$.

3[(4-chloro, 2-methyl phenyl)-5-methyl-1acetylamino)]-1,3,4-triazole-2-ones (IIIb) IR $\left(\mathrm{KBr}, \mathrm{cm}^{-1}\right): 1682(>\mathrm{C}=\mathrm{O}$ Stretching of side chain), 1726 (ring $>\mathrm{C}=\mathrm{O}$ Stretching) \& 3055 $\mathrm{cm}^{-1}$ (NH stretching). ${ }^{1} \mathrm{H}$ NMR (DMSO-d $\left.{ }_{6}\right) \delta: 6.2-8.8$ (m, 3H, Ar-H), 2.20 (s, 3H, N-CH ${ }_{3}$ ), $5.48\left(\mathrm{~s}, 3 \mathrm{H}, \mathrm{CH}_{3}\right), 8.4(\mathrm{~s}, 1 \mathrm{H},-\mathrm{NH})$. Mass spectra $(\mathrm{m} / \mathrm{z}): \mathrm{M}^{+1}=280$. Elemental data cal, $\mathrm{C}$, 51.34; H, 4.67; Cl, 12.63; N, 19.96; O, 11.40. Found: C, 51.22; H, 4.58; Cl, 12.58; N, 19.84; O, 11.44. Yield: $59 \%$. MP: $295-297{ }^{\circ} \mathrm{C}$.

3[(4-bromo, 2-methyl phenyl)-5-methyl-1acetylamino)]-1,3,4-triazole-2-ones (IIIc) $\mathrm{IR}\left(\mathrm{KBr}, \mathrm{cm}^{-1}\right): 1682(>\mathrm{C}=\mathrm{O}$ Stretching of side chain), 1726 (ring $>\mathrm{C}=\mathrm{O}$ Stretching) \& 3055 $\mathrm{cm}^{-1}$ (NH stretching). ${ }^{1} \mathrm{H}$ NMR (DMSO-d $\left.\mathrm{d}_{6}\right) \delta: 6.1-8.7$ (m, 3H, Ar-H), $2.20\left(\mathrm{~s}, 3 \mathrm{H}, \mathrm{N}-\mathrm{CH}_{3}\right)$, $5.48\left(\mathrm{~s}, 3 \mathrm{H}, \mathrm{CH}_{3}\right), 8.4(\mathrm{~s}, 1 \mathrm{H},-\mathrm{NH})$. Mass spectra $(\mathrm{m} / \mathrm{z}): \mathrm{M}^{+1}=324$. Elemental data cal, $\mathrm{C}$, 44.33; H, 4.03; Br, 24.57; N, 17.23; O, 9.84. Found: C, 44.23; H, 4.13; Br, 24.48; N, 17.16; O, 9.78. Yield: $45 \%$. MP: $213-215^{\circ} \mathrm{C}$.

3[(phenyl)-5-methyl-1acetylamino)]-1,3,4-triazole-2-ones (IIId)

IR $\left(\mathrm{KBr}, \mathrm{cm}^{-1}\right): 1682(>\mathrm{C}=\mathrm{O}$ Stretching of side chain), 1726 ( ring $>\mathrm{C}=\mathrm{O}$ Stretching) \& 3055 $\mathrm{cm}^{-1}$ (NH stretching). ${ }^{1} \mathrm{H}$ NMR (DMSO-d $\left.{ }_{6}\right) \delta: 6.1-8.7(\mathrm{~m}, 5 \mathrm{H}, \mathrm{Ar}-\mathrm{H}), 2.20\left(\mathrm{~s}, 3 \mathrm{H}, \mathrm{N}-\mathrm{CH}_{3}\right)$, $8.2(\mathrm{~s}, 1 \mathrm{H},-\mathrm{NH})$. Mass spectra $(\mathrm{m} / \mathrm{z}): \mathrm{M}^{+1}=232$. Elemental data cal, $\mathrm{C}, 56.89 ; \mathrm{H}, 5.21 ; \mathrm{N}$, 24.12; O, 13.78. Found: C, 56.78; H, 5.24; N, 24.08; O, 13.69. Yield: $41 \%$. MP: $330-332{ }^{\circ} \mathrm{C}$.

3[(4-methoxy phenyl)-5-methyl-1acetylamino)]-1,3,4-triazole-2-ones (IIIe)

IR $\left(\mathrm{KBr}, \mathrm{cm}^{-1}\right)$ : $1680(>\mathrm{C}=\mathrm{O}$ Stretching of side chain), 1722 (ring $>\mathrm{C}=\mathrm{O}$ Stretching) \& 3045 $\mathrm{cm}^{-1}$ (NH stretching). ${ }^{1} \mathrm{H}$ NMR (DMSO-d $\left.\mathrm{d}_{6}\right) \delta: 6.6-8.2(\mathrm{~m}, 4 \mathrm{H}, \mathrm{Ar}-\mathrm{H}), 2.20\left(\mathrm{~s}, 3 \mathrm{H}, \mathrm{N}-\mathrm{CH}_{3}\right)$, $3.68\left(\mathrm{~s}, 3 \mathrm{H}, 4 \mathrm{OCH}_{3}\right), 8.2(\mathrm{~s}, 1 \mathrm{H},-\mathrm{NH})$. Mass spectra $(\mathrm{m} / \mathrm{z}): \mathrm{M}^{+1}=246$. Elemental data cal, C, 58.53; H, 5.73; N, 22.75; O, 12.99. Found: C, 58.46; H, 5.68; N, 22.72; O, 12.87 Yield: 51\%. MP: $301-303{ }^{\circ} \mathrm{C}$.

3[(2,4-dimethoxy phenyl)-5-methyl-1acetylamino)]-1,3,4-triazole-2-ones (IIIf)

IR $\left(\mathrm{KBr}, \mathrm{cm}^{-1}\right): 1680(>\mathrm{C}=\mathrm{O}$ Stretching of side chain), 1722 (ring $>\mathrm{C}=\mathrm{O}$ Stretching) \& 3045 $\mathrm{cm}^{-1}$ (NH stretching). ${ }^{1} \mathrm{H}$ NMR (DMSO-d $\left.{ }_{6}\right) \delta: 6.6-8.2(\mathrm{~m}, 4 \mathrm{H}, \mathrm{Ar}-\mathrm{H}), 2.20\left(\mathrm{~s}, 3 \mathrm{H}, \mathrm{N}-\mathrm{CH}_{3}\right.$ ), $3.68\left(\mathrm{~s}, 3 \mathrm{H}, 4 \mathrm{OCH}_{3}\right), 8.2(\mathrm{~s}, 1 \mathrm{H},-\mathrm{NH})$. Mass spectra $(\mathrm{m} / \mathrm{z}): \mathrm{M}^{+1}=292$. Elemental data cal, $\mathrm{C}$, 53.42; H, 5.52; N, 19.17; O, 21.90. Found: C, 53.44; H, 5.50; N, 19.14; O, 21.82. Yield: 65\%. MP: $245-247^{\circ} \mathrm{C}$.

\section{Results and Discussion}

Biological activity:

All the newly synthesized compounds were screened for their antimicrobial activity by cup plate method at $100 \mu \mathrm{g} / \mathrm{ml}$ concentration in DMF against the Bacterial strains viz., E. Coli \& B. Subtilis and also against Fungal strains viz., A Niger and A. Sereus. Norfloxacin for bacteria and Griseofulvin as the reference drugs respectively. All these compounds were less 
active against the bacterial strains, but some of them showed selective fungal inhibitory activity (Table-4).

Table 4. Anti-microbial activities of synthesized compounds I(a-f), II(a-f), and III(a-f).

\begin{tabular}{ccccc}
\hline $\begin{array}{c}\text { Compound } \\
\text { Code }\end{array}$ & E.coli & B. subtilis & A. niger & C. Albicans \\
\hline Ia & 12 & 11 & 18 & 17 \\
Ib & 13 & 14 & 22 & 21 \\
Ic & 14 & 15 & 21 & 19 \\
Id & 15 & 14 & 20 & 21 \\
Ie & 13 & 14 & 21 & 20 \\
If & 13 & 13 & 18 & 17 \\
IIa & 14 & 12 & 20 & 18 \\
IIb & 13 & 12 & 18 & 16 \\
IIc & 16 & 14 & 20 & 21 \\
IId & 13 & 12 & 19 & 22 \\
IIe & 08 & 12 & 16 & 18 \\
IIf & 10 & 13 & 14 & 12 \\
IIIa & 12 & 11 & 22 & 16 \\
IIIb & 10 & 12 & 18 & 15 \\
IIIc & 14 & 12 & 26 & 21 \\
IIId & 11 & 10 & 12 & 14 \\
IIIe & 12 & 14 & 14 & 11 \\
IIIf & 10 & 16 & 16 & 18 \\
Norfloxacin & 22 & 22 & -- & -- \\
Grisiofulvin & -- & -- & 24 & 04 \\
DMF & 04 & 04 & 04 & \\
\hline & & & & 24 \\
\hline
\end{tabular}

\section{Acknowledgments}

We are thankful to USIC Dharwad for providing the spectral studies, authorities of BioGenics, Hubli for providing facilities in carrying out the biological studies of the compounds.

We express our deep sense of gratitude to Principal, Karnatak Science College, Dharwad, Director, USIC, KUD, the KLE's P.C. Jabin Science College, Hubli for providing the necessary facilities to carry out the research work.

\section{References:}

1. Kotresh O, Badami B V Indian J Het Chem., 2005, 15, 55-58.

2. Singh G B and Singh S, Indian Drugs, 1990, 27, 449-455.

3. Reddy A M, Reddy R R and Reddy VM, Indian J Pharm Sci., 1991, 53, 229-232.

4. Chaurasia M R, Sharma S K and Kumar R, Agri Boil Chem., 1980, 44, 663; Chem Abstr., 1980, 93, 4658.

5. Gupta D P, Ahmed S, Kumar A and SHanker K, Indian J Chem., 1998, 27B, 1060-1062. 
6. Shirodkar P Y and Meghna M Vartak, Indian J Het Chem., 2000, 9, 319- 320.

7. Shawkat S, Sudhir Singh and Satyavan Sharma, Indian J Chem., 1991, 30 B, 494- 498.

8. Rao A D, Ravi Shanker Ch, Reddy V M, Deshpande N and Venkat Rao Y, India Pharm Sci., 1986, 48(1), 13-15.

9. Achaiah G, Jayamma Y and Reddy V M, Indian J Het Chem., 1991, 1, 39-42.

10. Kavali J R, Kotresh O and Badami B V, J Chem Res (S)., 2003, 275.

11. Sari, N.; Arslan, S.; Logoglu, E.; Sakiyan, L. Antibacterial Activities of some Aminoacid-Schiff bases. GUJ Sci. 2003, 16, 283-288.

12. M D Salahuddin et al. E-Journal of Chemistry, 2009, 6(4), 10551062 . 


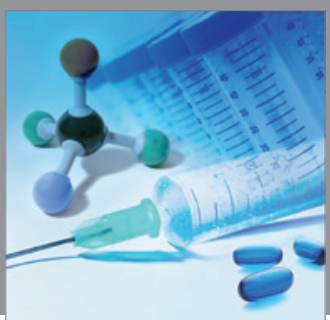

International Journal of

Medicinal Chemistry

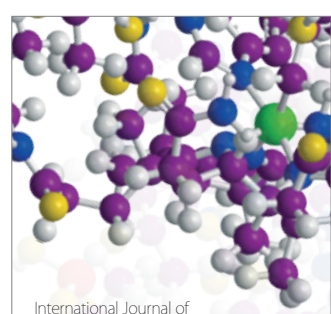

Carbohydrate Chemistry

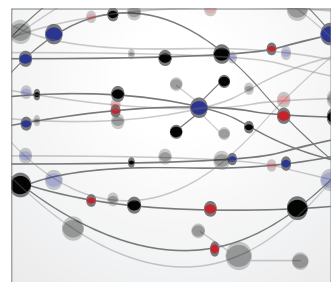

The Scientific World Journal
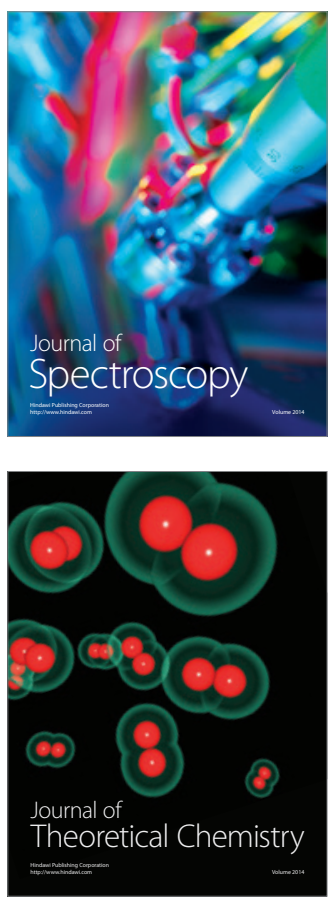
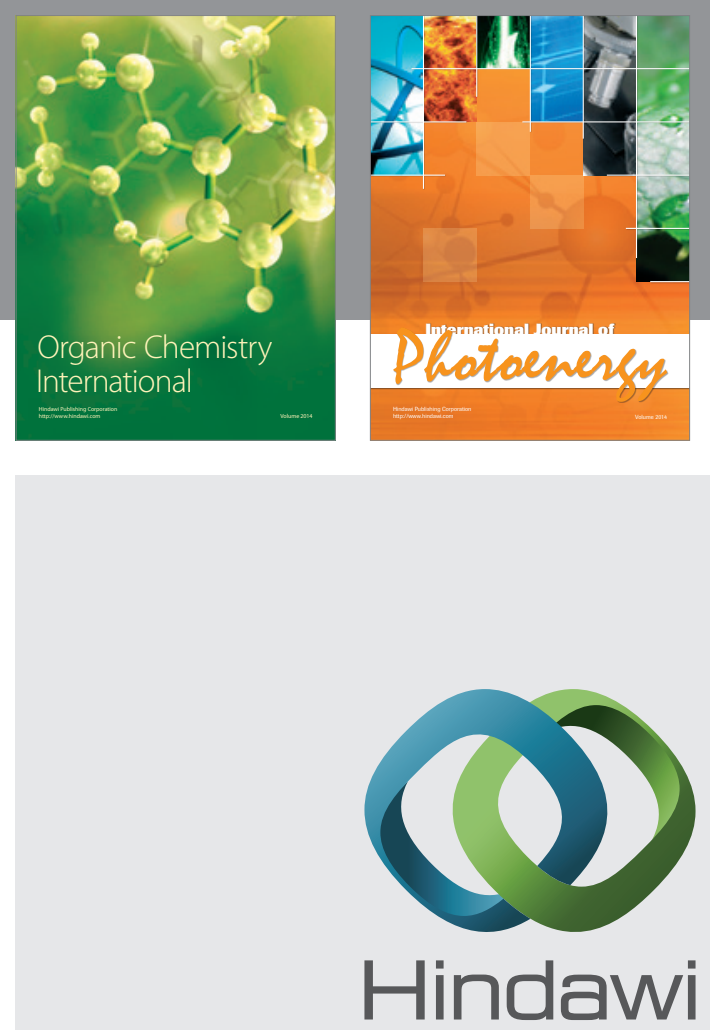

Submit your manuscripts at

http://www.hindawi.com
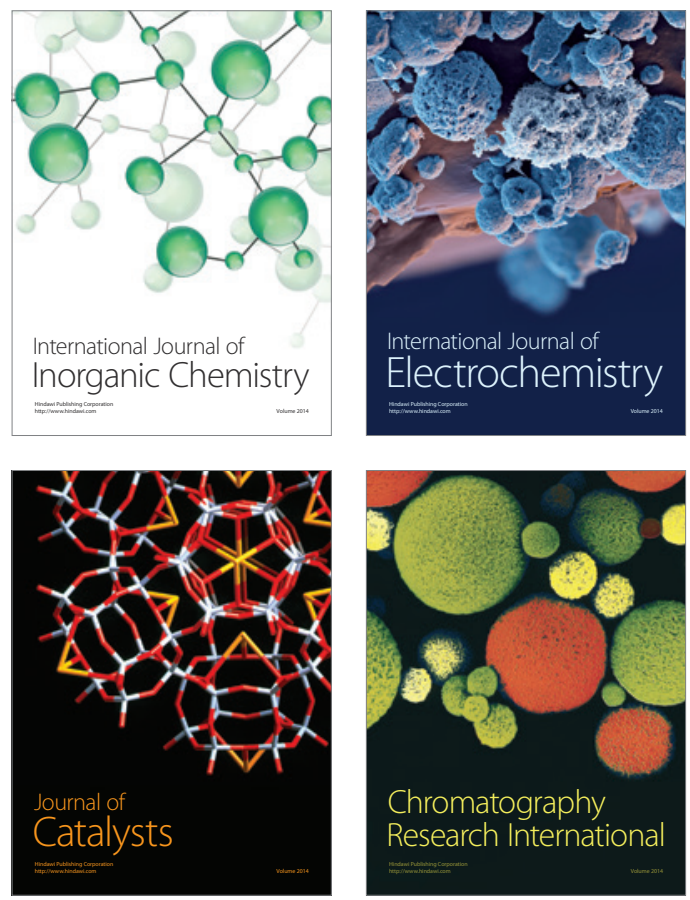
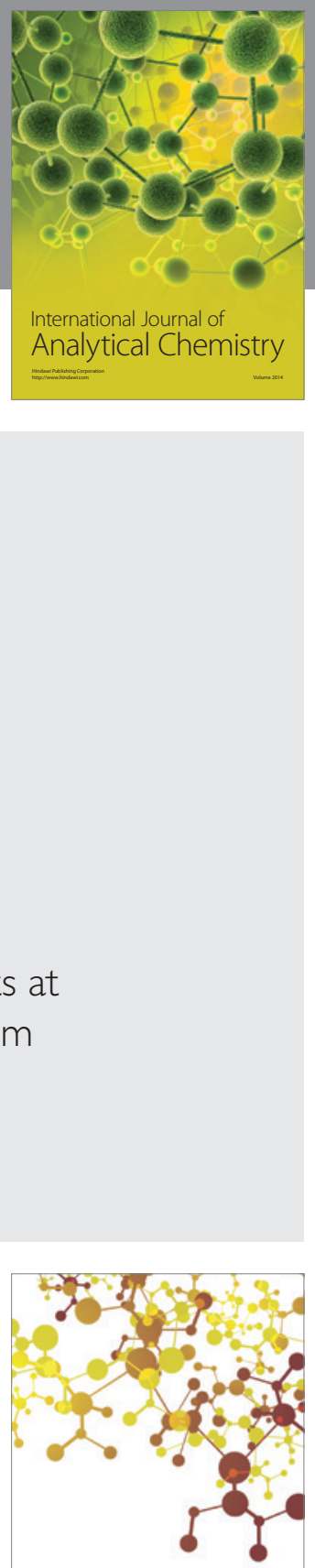

Journal of

Applied Chemistry
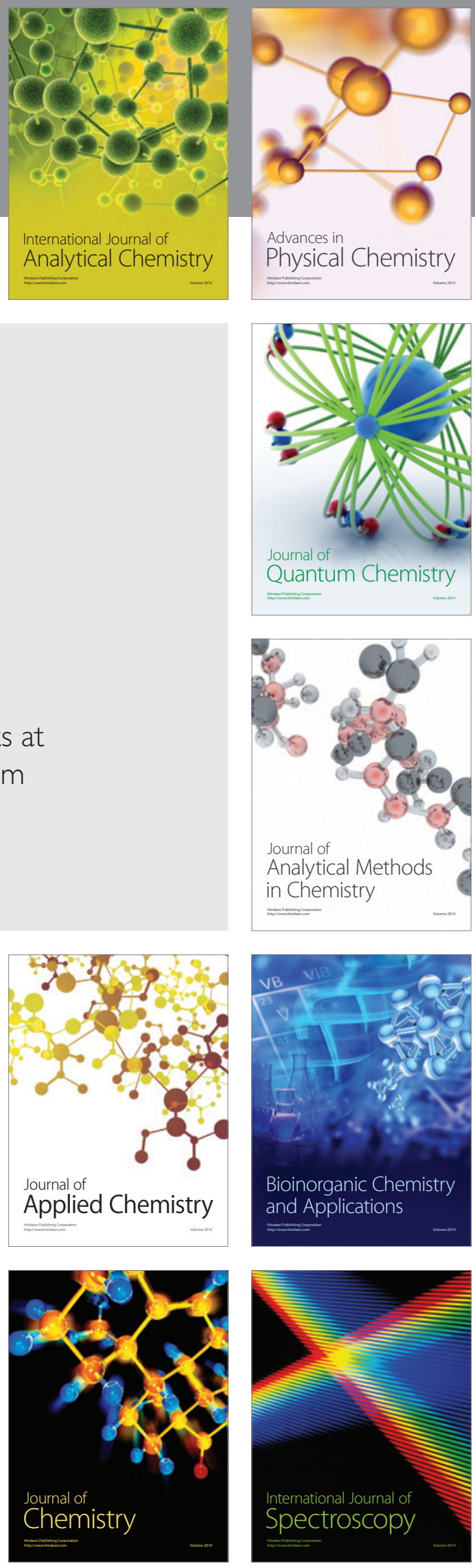